\title{
Early postnatal docosahexaenoic acid levels and improved preterm brain development
}

\author{
Emily W.Y.Tam', Vann Chau', A. James Barkovich", 2,3, , Donna M. Ferriero ${ }^{2,3}$, Steven P. Miller, ${ }^{1,5}$, Elizabeth E. Rogers ${ }^{3}$, \\ Ruth E. Grunau ${ }^{5}$, Anne R. Synnes ${ }^{5}$, Duan Xu ${ }^{4}$, Justin Foong ${ }^{1,6}$, Rollin Brant ${ }^{7}$ and Sheila M. Innis ${ }^{5}$
}

BACKGROUND: Preterm birth has a dramatic impact on polyunsaturated fatty acid exposures for the developing brain. This study examined the association between postnatal fatty acid levels and measures of brain injury and development, as well as outcomes.

METHODS: A cohort of 60 preterm newborns (24-32wk gestational age) was assessed using early and near-term magnetic resonance imaging (MRI) studies. Red blood cell fatty acid composition was analyzed coordinated with each scan. Outcome at a mean of 33 mo corrected age was assessed using the Bayley Scales of Infant Development, 3rd edition.

RESULTS: Adjusting for confounders, a 1\% increase in postnatal docosahexaenoic acid (DHA) levels at early MRI was associated with 4.3-fold decreased odds of intraventricular hemorrhage, but was not associated with white matter injury or cerebellar haemorrhage. Higher DHA and lower linoleic acid (LA) levels at early MRI were associated with lower diffusivity in white matter tracts and corresponding improved developmental scores in follow-up.

CONCLUSION: Higher DHA and lower LA levels in the first few weeks of life are associated with decreased intraventricular haemorrhage, improved microstructural brain development, and improved outcomes in preterm born children. Early and possibly antenatal interventions in high-risk pregnancies need to be studied for potential benefits in preterm developmental outcomes.

\section{$\mathbf{P}$} reterm birth is associated with increased risk for adverse neurodevelopmental impairment in survivors. Brain imaging has provided many clues as to the mechanisms of impaired outcomes and brain injury patterns such as intraventricular haemorrhage (IVH) $(1,2)$, white matter injury (WMI) $(3,4)$, and cerebellar haemorrhage $(5,6)$ have been shown to be predictive of motor and cognitive deficits. Advanced neuroimaging techniques such as diffusion tensor imaging provide tools to study brain microstructural development (7). These methods have enabled our understanding of risk factors for impaired brain development, such as infection (4).

Preterm birth, with premature transition to parenteral nutrition, abruptly changes the nutritional exposures of the developing brain. Placental transfer of fatty acids in the third trimester is low, about $10 \%$ of total energy, but comprises a much higher proportion of long-chain polyunsaturated fatty acid (PUFA) than in maternal plasma (8). Docosahexaenoic acid (DHA), a key essential PUFA for the nervous system, falls to one-third whole blood in utero levels within $2-3 \mathrm{wk}$ after preterm birth (9). Even late preterm birth is associated with significant disruption of normal essential PUFA levels, including lower $\omega$-3 fatty acid levels (10). Essential PUFAs cannot be synthesized de novo, but are important components of cell membranes. Notably, DHA may play a role in decreased brain injury $(11,12)$ and improved development (13-15). In addition, the $\omega-6$ PUFA arachidonic acid (ARA) is a precursor to hormones that regulate inflammation, including prostaglandins and leukotrienes, with additional potential effects.

Although metaanalyses have not shown overall developmental improvements with postnatal supplementation of DHA and ARA (16), specific early benefits of DHA with or without ARA have been demonstrated in visual function (17) and some cognitive tests (18-21). One limitation of these prior studies is the variability in dosage and timing of interventions. Few studies have looked at specific neuroimaging markers for the neurodevelopmental effects of PUFAs to determine the appropriate timing and targeting of intervention to effect improvements. This study aims to assess the associations between early postnatal red blood cell PUFA levels and signs of brain injury (including IVH, WMI, and cerebellar haemorrhage) and development using serial magnetic resonance imaging (MRI) studies. It is hypothesized that higher levels of $\omega-3$ fatty acids, specifically DHA, would be associated with decreased risk for brain injury and improved brain development and neurodevelopmental outcomes in infants born very preterm.

\footnotetext{
${ }^{1}$ Department of Pediatrics, Hospital for Sick Children and University of Toronto, Toronto, Ontario, Canada; ${ }^{2}$ Department of Neurology, University of California San Francisco, San Francisco, California; ${ }^{3}$ Department of Pediatrics, University of California San Francisco, San Francisco, California; ${ }^{4}$ Department of Radiology \& Diagnostic Imaging, University of California San Francisco, San Francisco, California; ${ }^{5}$ Department of Pediatrics, University of British Columbia, Vancouver, British Columbia, Canada; ${ }^{6} \mathrm{Centre}$ for Computational Medicine, Hospital for Sick Children, Toronto, Ontario, Canada; ${ }^{7}$ Department of Statistics, University of British Columbia, Vancouver, BC, Canada. Correspondence: Emily W.Y. Tam (emily.tam@utoronto.ca)

Received 11 September 2015; accepted 12 November 2015; advance online publication 3 February 2016. doi:10.1038/pr.2016.11
} 


\section{Articles $\mid$ Tametal.}

\section{RESULTS}

A total of 60 preterm newborns $<32 \mathrm{wk}$ gestational age (GA) were enrolled from the two study centers (Table 1). All newborns completed early MRI scan (mean $31.54 \pm 2.26 \mathrm{wk} \mathrm{GA}$ ) and associated blood sampling, with 44/60 (73\%) completing the near-term age MRI scan (mean $37.71 \pm 2.98$ wk GA) and associated blood sampling. Infants who were discharged home prior to term and unable to return for outpatient testing were missed. Early blood sample showed a median DHA level of 4.4\% (range 2-6.4\%), eicosapentaenoic acid (EPA) level of $0.3 \%$ (range $0.1-4 \%$ ), ARA level of $16.4 \%$ (range 10.8-20.4\%), and linoleic acid (LA) level of 9.2\% (range 5.9-14.1\%). Near-term age samples showed a median DHA level of $4.5 \%$ (range $0.7-7.7 \%$ ), EPA level of $0.4 \%$ (range $0.1-0.8 \%)$, ARA level of $16.6 \%$ (3.7-19.2\%), and LA level of $10.4 \%$ (range $6.2-14.5 \%$ ).

Table 1. Patient demographics at UCSF and UBC

\begin{tabular}{|c|c|c|}
\hline & $\operatorname{UCSF}(N=33)$ & $\mathrm{UBC}(N=27)$ \\
\hline Gestational age at birth (wk) & $28.05 \pm 1.75$ & $28.49 \pm 2.03$ \\
\hline 5-min Apgar score & $8($ IQR 7-8) & $8($ IQR 6-8) \\
\hline Umbilical artery $\mathrm{pH}$ & $7.26 \pm 0.11$ & $7.44 \pm 1.33$ \\
\hline Male sex & $20(61 \%)$ & $17(63 \%)$ \\
\hline $\begin{array}{l}\text { Antenatal glucocorticoid } \\
\text { exposure (any) }\end{array}$ & $33(100 \%)$ & $17(63 \%)$ \\
\hline Clinical chorioamnionitis & $5(15 \%)$ & $5(19 \%)$ \\
\hline Neonatal sepsis & $17(52 \%)$ & $18(67 \%)$ \\
\hline Patent ductus arteriosus & $16(48 \%)$ & $10(37 \%)$ \\
\hline Hypotension & $21(64 \%)$ & $21(78 \%)$ \\
\hline Intubation (d) & $11.64 \pm 26.10$ & $10.70 \pm 20.25$ \\
\hline Parenteral nutrition (d) & $15.45 \pm 8.17$ & $14.67 \pm 10.39$ \\
\hline Intralipid supplementation (d) & $13.61 \pm 7.93$ & $14.15 \pm 10.45$ \\
\hline \multicolumn{3}{|l|}{ Enteral nutrition } \\
\hline $\begin{array}{l}\text { Breast milk }(\mathrm{d}) / \text { total days } \\
\text { from birth to near-term blood } \\
\text { sample }\end{array}$ & 78\% (IQR 63-92\%) & 96\% (IQR 70-99\%) \\
\hline $\begin{array}{l}\text { Formula }(\mathrm{d}) / \text { total days from } \\
\text { birth to near-term blood sample }\end{array}$ & $12 \%($ IQR $0-57 \%)$ & 0\% (IQR 0-43\%) \\
\hline Postmenstrual age at first MRI (wk) & $31.04 \pm 1.65$ & $32.14 \pm 2.75$ \\
\hline $\begin{array}{l}\text { Postmenstrual age at second } \\
\text { MRI (wk) }\end{array}$ & $36.13 \pm 2.42$ & $40.22 \pm 1.85$ \\
\hline \multicolumn{3}{|l|}{ Intraventricular hemorrhage } \\
\hline Mild (grade 1-2) & $4(12 \%)$ & $5(19 \%)$ \\
\hline Severe (grade 3-4) & $6(18 \%)$ & $1(4 \%)$ \\
\hline \multicolumn{3}{|l|}{ White matter injury } \\
\hline Mild & $2(6 \%)$ & $5(19 \%)$ \\
\hline Moderate/severe & $6(18 \%)$ & $3(11 \%)$ \\
\hline Cerebellar hemorrhage & $4(12 \%)$ & $2(7 \%)$ \\
\hline
\end{tabular}

Expressed as mean $\pm S D$, mean and intraquartile range (IQR), or frequency and percentage.

UCSF, University of California San Francisco; UBC, University of British Columbia; MRI, magnetic resonance imaging.

\section{PUFAs and Brain Injury on Conventional MRI Scanning}

Fatty acid levels were compared with findings of IVH, WMI, and cerebellar hemorrhage. Univariate logistic regression analysis showed trends to association with IVH for DHA (odds ratio $0.48,95 \% \mathrm{CI} 0.22-1.06, P=0.070$ ) and ARA (odds ratio $0.77,95 \%$ CI $0.56-1.05, P=0.098$ ), but no other fatty acids and WMI or cerebellar hemorrhage $(P>0.3)$. After adjusting for confounders, only DHA remained significantly associated with IVH (Table 2). One percent increase in DHA was associated with a 4.3 -fold decreased odds of IVH $(P=0.024)$. Of note, brain injury scores did not change from first to second scan, however no association was found between near-term fatty acid levels and brain injury patterns including IVH, WMI, or cerebellar haemorrhage $(P>0.2)$.

Lower DHA levels were associated with higher IVH severity (Figure 1). Using ordinal logistic regression analysis, 1\% increase in DHA was associated with a 2.3 -fold decreased odds of increasing one degree of IVH severity (95\% CI 1.065.27, $P=0.036$ ), when IVH was categorized as none, mild (grade 1-2), or severe (grade 3-4). Adjusting for confounders, $1 \%$ increase DHA was associated with 3.9-fold decreased odds of increasing one degree of IVH severity (95\% CI 1.22-12.28, $P=0.022)$.

Table 2. Logistic regression analysis comparing red blood cell fatty acid levels (percent total fatty acid) and presence of brain injury (of all severity grades) on testing as soon after birth as clinically stable for MRI scanning

\begin{tabular}{|c|c|c|c|}
\hline & OR & $95 \% \mathrm{Cl}$ & $P$ value \\
\hline \multicolumn{4}{|l|}{ DHA, 22:6ஸ3 } \\
\hline IVH & 0.23 & $0.06-0.83$ & 0.02 \\
\hline WMI & 0.83 & $0.24-2.86$ & 0.76 \\
\hline Cerebellar hemorrhage & 0.69 & $0.10-5.06$ & 0.72 \\
\hline \multicolumn{4}{|l|}{ EPA, 20:5 $\omega 3$} \\
\hline IVH & 2.68 & $0.38-18.93$ & 0.32 \\
\hline WMI & 0.17 & $0.01-3.66$ & 0.26 \\
\hline Cerebellar hemorrhage & 21.72 & $0.02-20652.18$ & 0.38 \\
\hline \multicolumn{4}{|l|}{ 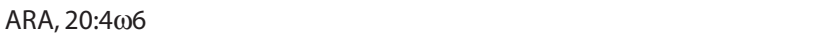 } \\
\hline IVH & 0.82 & $0.58-1.16$ & 0.26 \\
\hline WMI & 0.88 & $0.59-1.30$ & 0.51 \\
\hline Cerebellar hemorrhage & 0.37 & $0.10-1.27$ & 0.11 \\
\hline \multicolumn{4}{|l|}{ LA, $18: 2 \omega 6$} \\
\hline IVH & 1.20 & $0.84-1.71$ & 0.31 \\
\hline WMI & 0.92 & $0.60-1.42$ & 0.71 \\
\hline Cerebellar hemorrhage & a & a & $\mathrm{a}$ \\
\hline
\end{tabular}

Results are adjusted for potential confounders, including gestational age at birth, chorioamnionitis, antenatal betamethasone exposure, 5-min Apgar score, patent ductus arteriosus, hypotension, intubation, and neonatal sepsis.

$\mathrm{MRI}$, magnetic resonance imaging; OR, optic radiations; DHA, docosahexaenoic acid; EPA, eicosapentaenoic acid; ARA, arachidonic acid; LA, linoleic acid; IVH, intraventricular haemorrhage; WMI, white matter injury.

aDenotes inadequate data for statistical analysis. 


\section{PUFAs and Brain Microstructure on DTI}

Using tract-based spatial statistics, fatty acid levels on early preterm and near-term age blood samples were compared with the diffusion tensor imaging measures of fractional anisotropy (FA), axial diffusivity, and radial diffusivity, adjusting for age at scan. No statistically significant associations were found for any fatty acid levels in the near-term age blood sample. In the early preterm blood sample, only DHA was found to be associated with axial and radial diffusivity (Figure 2). No association was found with FA.

To compliment these tract-based spatial statistics findings, regions of interest were drawn in the posterior limbs of the internal capsule (PLIC) and the optic radiations (OR), both functionally relevant pathways, as indicated in blue in Figure 2. Diffusion measures in both time points were compared with early preterm fatty acid levels. Results after adjusting for clinical confounders are described in Table 3. Of note, higher DHA levels were inversely associated with mean diffusivity in the PLIC and OR, and axial diffusivity in the PLIC. Higher LA levels were associated with higher mean diffusivity in the OR.

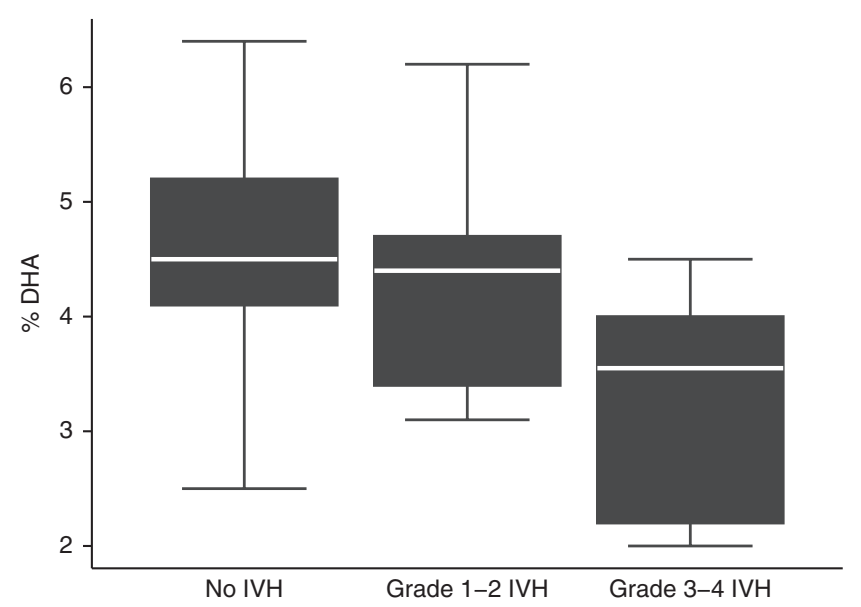

Figure 1. Percent red blood cell DHA and IVH. Boxplot is divided into infants with no, mild (grade 1-2), or severe (grade 3-4) IVH. DHA, docosahexaenoic acid; IVH, intraventricular haemorrhage.
Due to the association between DHA and IVH, subanalysis was performed excluding infants with grade 3-4 IVH (Table 4). Excluding these infants did not meaningfully change the associations found in the PLIC, but did strengthen the associations found in the OR, with additional statistically significant association seen between higher DHA levels and lower radial diffusivity. As in prior analyses, no statistically significant associations were found with EPA or ARA. However after excluding infants with grade 3-4 IVH, LA no longer had a statistically significant association with mean diffusivity in the OR.

\section{PUFAs and Postnatal Nutrition}

Table 5 details the comparisons between fatty acid levels and postnatal nutritional exposures prior to each blood sample. Since intravenous nutrition and lipid supplementation were completed prior to the first scans and blood samples, the number of days of these interventions were compared with the early samples. Since the number of days between birth and near-term testing varied significantly between subjects of varied GA at birth, the percent of days exposed to breast milk or formula was compared with near-term samples. There was a trend to lower early DHA levels with increased duration of IV nutrition, however no significant associations were found between early and near-term DHA levels and nutrition modalities. Duration of intravenous nutrition was associated with higher early EPA and LA levels, and lower early ARA levels. Percentage of days of formula feeding was associated with lower term EPA levels.

\section{PUFAs and Developmental Outcome}

Developmental assessments were performed on 25 (76\%) of the enrolled children at UCSF and 20 (74\%) at UBC. Assessment was at a mean of $32.8 \pm 3.6$ mo corrected age. There was no difference in fatty acid levels between those assessed in follow-up and those lost to follow-up $(P>0.09)$. Mean Bayley-3 cognitive scores were 103.6 \pm 14.4 , language scores $101.0 \pm 17.8$, and motor scores $98.1 \pm 14.3$. Although there was no association between early DHA levels and Bayley-3 cognitive scores $(P=$ 0.4 ), a $1 \%$ increase in early DHA level was associated with 7.0

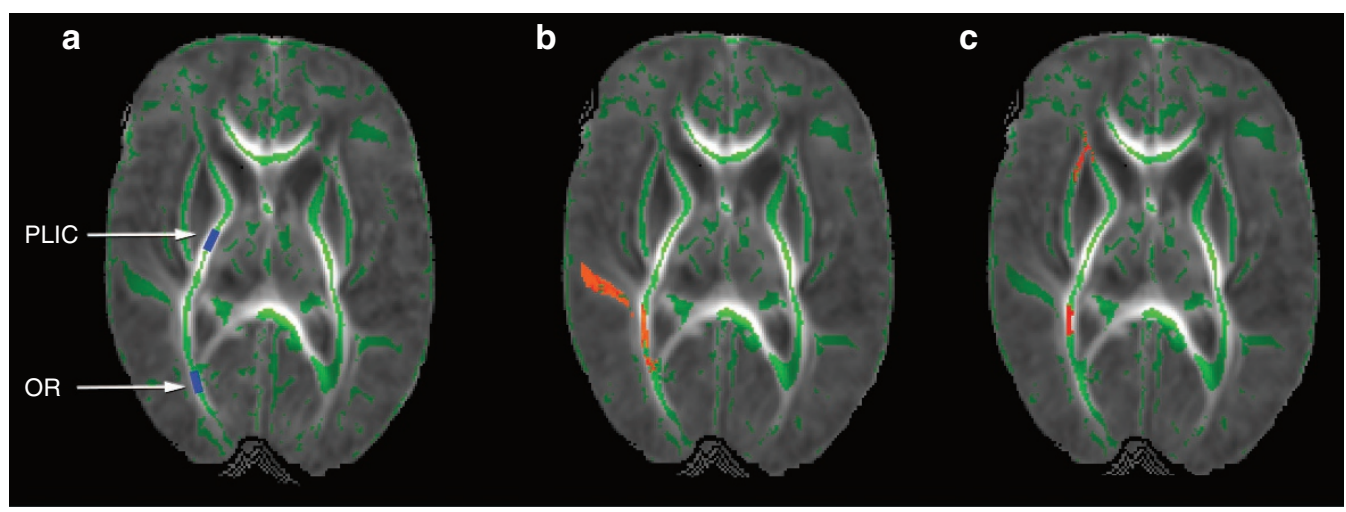

Figure 2. Axial fractional anisotropy maps showing the white matter skeleton in green. Regions of the white matter skeleton showing significant associations between early preterm red blood cell docosahexaenoic acid levels and (a) fractional anisotropy, (b) axial diffusivity, and (c) radial diffusivity are highlighted in shades ranging from yellow $(P=0.05)$ to red $(P=0.00)$. Regions of interest in the PLIC and OR are marked in blue. PLIC, posterior limbs of the internal capsule; OR, optic radiations. 


\section{Articles | Tametal.}

Table 3. Linear mixed regression analysis comparing early preterm red blood cell fatty acid levels (percent total fatty acid) and diffusion tensor imaging measures on serial MRI scanning in the PLIC and the OR

\begin{tabular}{|c|c|c|c|c|c|c|}
\hline & \multicolumn{3}{|c|}{ PLIC } & \multicolumn{3}{|c|}{ OR } \\
\hline & Change & $95 \% \mathrm{Cl}$ & $P$ value & Change & $95 \% \mathrm{Cl}$ & $P$ value \\
\hline \multicolumn{7}{|l|}{ DHA, 22:6ஸ3 } \\
\hline Fractional anisotropy & -0.0021 & -0.0218 to 0.0176 & 0.83 & 0.0106 & -0.0160 to 0.0372 & 0.44 \\
\hline Axial diffusivity $\left(10^{-3} \mathrm{~mm}^{2} / \mathrm{s}\right)$ & -0.0449 & -0.0787 to -0.0111 & 0.01 & -0.0477 & -0.1115 to 0.0161 & 0.14 \\
\hline \multicolumn{7}{|l|}{ EPA, 20:5 $\omega 3$} \\
\hline Mean diffusivity $\left(10^{-3} \mathrm{~mm}^{2} / \mathrm{s}\right)$ & -0.0039 & -0.1529 to 0.1451 & 0.96 & -0.0339 & -0.2614 to 0.1937 & 0.77 \\
\hline Fractional anisotropy & -0.0638 & -0.1603 to 0.0328 & 0.20 & 0.1279 & -0.0050 to 0.2608 & 0.06 \\
\hline Axial diffusivity $\left(10^{-3} \mathrm{~mm}^{2} / \mathrm{s}\right)$ & -0.0881 & -0.2679 to 0.0918 & 0.34 & 0.1631 & -0.1717 to 0.4979 & 0.34 \\
\hline Radial diffusivity $\left(10^{-3} \mathrm{~mm}^{2} / \mathrm{s}\right)$ & 0.0366 & -0.1307 to 0.2040 & 0.67 & -0.1285 & -0.3937 to 0.1367 & 0.34 \\
\hline Axial diffusivity $\left(10^{-3} \mathrm{~mm}^{2} / \mathrm{s}\right)$ & -0.0077 & -0.0227 to 0.0074 & 0.32 & -0.0049 & -0.0325 to 0.0227 & 0.73 \\
\hline Radial diffusivity $\left(10^{-3} \mathrm{~mm}^{2} / \mathrm{s}\right)$ & -0.0043 & -0.0183 to 0.0097 & 0.55 & -0.0014 & -0.0236 to 0.0208 & 0.90 \\
\hline \multicolumn{7}{|l|}{ LA, $18: 2 \omega 6$} \\
\hline Mean diffusivity $\left(10^{-3} \mathrm{~mm}^{2} / \mathrm{s}\right)$ & 0.0086 & -0.0016 to 0.0189 & 0.10 & 0.0162 & 0.0005 to 0.0319 & 0.04 \\
\hline Fractional anisotropy & -0.0033 & -0.0103 to 0.0038 & 0.36 & -0.0037 & -0.0137 to 0.0064 & 0.48 \\
\hline Axial diffusivity $\left(10^{-3} \mathrm{~mm}^{2} / \mathrm{s}\right)$ & 0.0060 & -0.0070 to 0.0189 & 0.37 & 0.0097 & -0.0147 to 0.0342 & 0.44 \\
\hline Radial diffusivity $\left(10^{-3} \mathrm{~mm}^{2} / \mathrm{s}\right)$ & 0.0100 & -0.0015 to 0.0215 & 0.09 & 0.0132 & -0.0056 to 0.0321 & 0.17 \\
\hline
\end{tabular}

Change values represent change in diffusion tensor imaging measures per $1 \%$ increase in red blood cell fatty acid level. Results are adjusted for postmenstrual age at time of each scan, IVH severity, WMI severity, sepsis, duration of intubation, and patent ductus arteriosus.

PLIC, posterior limbs of the internal capsule; OR, optic radiations; DHA, docosahexaenoic acid; EPA, eicosapentaenoic acid; ARA, arachidonic acid; LA, linoleic acid; MRI, magnetic resonance imaging.

Table 4. Linear mixed regression analysis comparing early preterm red blood cell DHA levels (percent total fatty acid) and diffusion tensor imaging measures on serial MRI scanning in the PLIC and the OR, excluding infants with grade 3-4 IVH

\begin{tabular}{|c|c|c|c|c|c|c|}
\hline & \multicolumn{3}{|c|}{ PLIC } & \multicolumn{3}{|c|}{ OR } \\
\hline & Change & $95 \% \mathrm{Cl}$ & $P$ value & Change & $95 \% \mathrm{Cl}$ & $P$ value \\
\hline Mean diffusivity $\left(10^{-3} \mathrm{~mm}^{2} / \mathrm{s}\right)$ & -0.0359 & -0.0663 to -0.0055 & 0.02 & -0.0747 & -0.1183 to -0.0311 & $<0.001$ \\
\hline Axial diffusivity $\left(10^{-3} \mathrm{~mm}^{2} / \mathrm{s}\right)$ & -0.0439 & -0.0813 to -0.0066 & 0.02 & -0.0609 & -0.1320 to 0.0102 & 0.09 \\
\hline Radial diffusivity $\left(10^{-3} \mathrm{~mm}^{2} / \mathrm{s}\right)$ & -0.0313 & -0.0667 to 0.0042 & 0.08 & -0.0642 & -0.1194 to 0.0090 & 0.02 \\
\hline
\end{tabular}

Change values represent change in diffusion tensor imaging measures per $1 \%$ increase in red blood cell DHA level. Results are adjusted for postmenstrual age at time of each scan, IVH severity, WMI severity, sepsis, duration of intubation, and patent ductus arteriosus.

PLIC, posterior limbs of the internal capsule; OR, optic radiations; DHA, docosahexaenoic acid; MRI, magnetic resonance imaging; IVH, intraventricular haemorrhage; WMI, white matter injury.

point higher language scores $(95 \% \mathrm{CI} 1.3-12.7, P=0.017)$ and 7.5 point higher motor scores $(95 \%$ CI 3.1-11.9, $P=0.001$ ). A $1 \%$ increase in early LA level was associated with a 2.4 -point decrease in cognitive scores (95\% CI 0.16-4.6, $P=0.036$ ) and 3.5-point decrease in motor scores ( $95 \%$ CI 1.3-5.6, $P=0.003$ ), but no association with language scores $(P=0.16)$. Only grade 3-4 IVH was found to be associated with lower Bayley-3 scores $(P<0.02)$. Adjusting for grade 3-4 IVH, early DHA levels continued to be associated with better motor scores $(4.8,95 \%$ CI $0.5-9.1, P=0.03$ ) and early LA levels associated with poorer motor scores $(-2.2,95 \% \mathrm{CI}-4.3$ to $-0.13, P=0.038)$. No associations were seen with cognitive or language scores. Early ARA and EPA levels were not associated with outcome $(P>$ 0.35).

Using univariate linear regression analysis to assess the contribution of socioeconomic status, early DHA levels were associated with maternal education $(0.08 \%$ per year, $95 \% \mathrm{CI}$ $0.0004-0.15, P=0.049)$, maternal age $(0.05 \%$ per year, $95 \% \mathrm{CI}$ $0.01-0.08, P=0.013)$, and Hispanic ethnicity $(-1.10,95 \% \mathrm{CI}$ -1.56 to $-0.65, P<0.001)$. Race was not associated with early 
Table 5. Change in early fatty acid levels compared with intravenous nutrition and late fatty acid levels compared with breast milk vs. formula feeding

\begin{tabular}{|c|c|c|c|}
\hline & $\%$ Change & $95 \% \mathrm{Cl}$ & $P$ value \\
\hline \multicolumn{4}{|c|}{ Early preterm red blood cell fatty acid levels } \\
\hline \multicolumn{4}{|l|}{$\mathrm{DHA}, 22: 6 \omega 3$} \\
\hline Days intravenous nutrition & $-2.19 \%$ & -4.62 to $0.25 \%$ & 0.08 \\
\hline Days lipid supplementation & $-1.62 \%$ & -4.06 to $0.82 \%$ & 0.19 \\
\hline \multicolumn{4}{|l|}{$E P A, 20: 5 \omega 3$} \\
\hline Days intravenous nutrition & $12.33 \%$ & $8.72-15.96 \%$ & $<0.001$ \\
\hline Days lipid supplementation & $12.57 \%$ & $9.07-16.07 \%$ & $<0.001$ \\
\hline \multicolumn{4}{|l|}{ 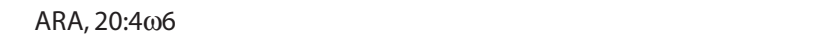 } \\
\hline Days intravenous nutrition & $-2.07 \%$ & -3.20 to $-0.93 \%$ & 0.001 \\
\hline Days lipid supplementation & $-2.00 \%$ & -3.13 to $-0.88 \%$ & 0.001 \\
\hline \multicolumn{4}{|l|}{ 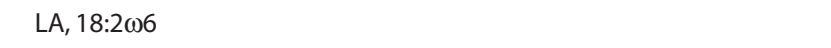 } \\
\hline Days intravenous nutrition & $1.96 \%$ & $0.85-3.06 \%$ & 0.001 \\
\hline Days lipid supplementation & $1.88 \%$ & $0.78-2.98 \%$ & 0.001 \\
\hline \multicolumn{4}{|c|}{ Near-term age red blood cell fatty acid levels } \\
\hline \multicolumn{4}{|l|}{$\mathrm{DHA}, 22: 6 \omega 3$} \\
\hline$\%$ Days breast milk & $-0.01 \%$ & -0.06 to $0.03 \%$ & 0.57 \\
\hline$\%$ Days formula & $-0.02 \%$ & -0.05 to $0.003 \%$ & 0.08 \\
\hline \multicolumn{4}{|l|}{$E P A, 20: 5 \omega 3$} \\
\hline$\%$ Days breast milk & $-0.04 \%$ & -0.35 to $0.27 \%$ & 0.80 \\
\hline$\%$ Days formula & $-0.19 \%$ & -0.37 to $-0.02 \%$ & 0.03 \\
\hline \multicolumn{4}{|l|}{ 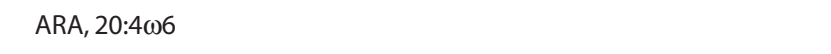 } \\
\hline$\%$ Days breast milk & $0.003 \%$ & -0.02 to $0.02 \%$ & 0.73 \\
\hline$\%$ Days formula & $0.003 \%$ & -0.01 to $0.01 \%$ & 0.57 \\
\hline \multicolumn{4}{|l|}{$L A, 18: 2 \omega 6$} \\
\hline$\%$ Days breast milk & $-0.01 \%$ & -0.04 to $0.03 \%$ & 0.72 \\
\hline \% Days formula & $-0.001 \%$ & -0.02 to $0.02 \%$ & 0.92 \\
\hline
\end{tabular}

Only nutrition from days prior to each blood sample was counted.

DHA, docosahexaenoic acid; EPA, eicosapentaenoic acid; ARA, arachidonic acid, LA, linoleic acid.

DHA levels $(P>0.17)$. No demographic variables were associated with early LA levels $(P>0.07)$. Using a backwards selection model to determine the contribution of each variable on outcomes, only DHA remained significantly associated with language scores $(7.0,95 \%$ CI 1.3-12.7, $P=0.017)$. However, both DHA $(6.7,95 \%$ CI 1.7-11.6, $P=0.01)$ and years of maternal education $(1.13,95 \%$ CI $0.03-2.2, P=0.044)$ were significantly associated with motor scores.

\section{DISCUSSION}

This study used serial blood samples, neuroimaging, and developmental assessment to present new evidence for an association between PUFA levels and preterm IVH and neurodevelopment. It has previously been suggested that $\omega$-3 fatty acid exposures, specifically DHA, may have positive effects on neurodevelopmental outcomes after preterm birth, yet the mechanisms of this protective effect have been unknown. Our study shows that higher early preterm red blood cell DHA levels are associated with lower incidence and severity of IVH. Measures of microstructural development suggest opposing associations with the $\omega-3$ fatty acid DHA and the $\omega-6$ fatty acid LA. Outcomes assessed at 30-36 mo further support these opposing associations.

Investigating the association between PUFA levels and brain injury, only early DHA levels were found to be associated with decreased incidence and severity of IVH. Although an association between PUFA levels and preterm brain injury has been previously postulated (12), there is currently no reported literature in humans or animal models for such an association.

Understanding the mechanisms for IVH after preterm birth is important for understanding the timing and potential mechanisms for an association with early DHA levels. Diagnosis of IVH usually occurs in the first day of life, and at least $90 \%$ of events are identified within the first $72 \mathrm{~h}$ (22). Thus, if DHA level was to have an effect on IVH incidence, it would have to be evident by the time the child was born, prior to hemorrhage. Accordingly, this study found that only the early DHA levels (collected on average of $3 \mathrm{wk}$ after birth) were associated with IVH.

DHA accounts for over $50 \%$ of all PUFAs in the central nervous system cell membranes (23). The specific molecular properties of this highly unsaturated fatty acid result in important changes in phase behavior, fluidity, permeability, and elasticity of cell membranes (24). Hemorrhage from IVH is believed to originate from the fragile capillary network of the subependymal germinal matrix, resulting from fluctuations in blood pressure and impaired cerebral autoregulation $(25,26)$. This highlights the importance of DHA in the central nervous system, and its potential effects on membrane elasticity and resistance to pressure changes that could affect the risk for haemorrhage in the preterm newborn.

Independent of IVH or WMI, early DHA levels were also found to be associated with long-term effects on white matter microstructural development. It has been previously shown in white matter tracts that axial and radial diffusivity decrease, while FA increases, with preterm brain development (27). Increases in FA may be partially related to increased premyelination and myelination, limiting radial mobility of water. Decreasing axial and radial diffusivity, however, may be due to decreasing brain water content and formation of new barriers to water mobility, including increased macromolecular concentration and outgrowth of neurons, glia, and blood vessels in all directions (28). Thus, the observed decrease in mean diffusivity with higher DHA and lower LA, without significant change in FA, suggests improved microstructural development independent of myelination. These findings are further supported by studies in macaque monkeys showing impairments in cortical network development after a lifetime of low dietary $\omega-3$ fatty acids (29).

Investigating the contribution of the neonatal feeding practices, although duration of parenteral nutrition had effects on EPA, LA, and ARA levels, no effects were seen with DHA levels. Lower EPA levels were seen with increased formula 
feeding, which is not surprising since Similac Special Care contains no EPA. Data collected in this study in regards to nutritional sources are limited to days exposed to each modality, and thus only estimates the exact exposures for each child. Finding lower EPA levels with formula feeding suggests that our estimated exposures would be able to detect trends in feeding differences. Our results suggest that the feeding regimens used during this study have no significant effect on postnatal DHA levels. Indeed, a Cochrane review of lipid supplementation showed no positive or negative association with outcomes (30).

This study also suggests that higher early DHA and lower early LA levels have long-term associations with improved developmental outcomes at 30-36 mo. These associations can be partially, but not fully explained by decreased rates of severe IVH. Markers of low socioeconomic status, including low maternal education, low maternal age, and Hispanic ethnicity were associated with lower early DHA levels, suggesting effects of socioeconomic status may begin antenatally, and not just after hospital discharge. Lack of association with race suggests that these differences are not as likely due to genetic variation. These results agree with prior studies showing a relationship between socioeconomic status and DHA levels in adults (31-33). This highlights the importance of the antenatal maternal diet on perinatal outcomes. Despite adjustment for socioeconomic status, however, early DHA and LA levels remained independently associated with outcomes, suggesting that in spite of potential ongoing differences in upbringing between these children, the early perinatal exposures remain an important determining factor.

The inclusion of two study sites enabled not only more rapid subject enrolment, but also an inclusion of a wider range of patient demographics and socioeconomic status. All analyses were adjusted for study site, and significant associations between fatty acid and brain metrics despite adjustment for illness severity suggests that these associations are independent of study site. It is interesting to note, however, that most of the Hispanic subjects in this study come from the UCSF study site, due to the demographics of San Francisco. With lower DHA levels associated with Hispanic ethnicity, it is thus not surprising based on current study findings that UCSF also demonstrated higher rates of IVH, even despite the potentially protective effects of antenatal glucocorticoid treatment at this study site.

For this study, red blood cell PUFA levels were used as a marker of overall fatty acid status. Compared with direct measurements of fatty acid levels within the brain tissue itself, red blood cell fatty acid levels are clearly less accurate. Red blood cell levels were assessed, rather than plasma levels, since plasma levels would be more dependent on day-to-day enteral and parenteral exposures in the neonatal period. However, red blood cell PUFAs are commonly used in animal and human studies as a marker for DHA and neurological development in infants $(17,34)$. In aged rats supplemented with DHA and EPA, good correlation has been found between red blood cell and brain DHA levels (35).
The results of this prospective observational study initially may seem to contradict prior studies of postnatal $\omega-3$ fatty acid supplementation, which did not show developmental benefits (16). However, our current findings may provide clues as to the appropriate timing and selection of targets for intervention. Higher early DHA levels are associated with lower rates and severity of IVH, while higher DHA and lower LA levels are associated with improved microstructural brain development and developmental outcomes at 30-36 mo. Since IVH occurs within $72 \mathrm{~h}$ of life, improvements in fatty acid profiles must be effected before this time, likely before birth. In addition, this study found lower early DHA levels to be associated with lower socioeconomic status, suggesting pre-existing and ongoing effects of maternal diet. Thus, further therapeutic trials will need to ensure early intervention and appropriate consideration for families with low socioeconomic status, to ensure appropriate targeting of vulnerable infants. Targeting of intervention and education to expectant mothers with low socioeconomic status may be the most effective for improving fatty acid levels and their implications on functional developmental outcomes.

\section{METHODS}

\section{Study Participants}

A prospective cohort of preterm newborns born between March 2010 and November 2011 admitted to the neonatal intensive care units at either the University of California San Francisco (UCSF) or the University of British Columbia (UBC) was studied. Parents of neonates born $<32$ wk GA were approached and consented following a protocol approved by research ethics board at both institutions. Infants were excluded if there was a congenital malformation or syndrome, congenital infection, or the infant was too clinically unstable to transport to the MRI scanner. Clinical history was collected prospectively from the patient charts. Exposure to parenteral nutrition, intravenous lipid supplementation, breast milk nutrition, and formula nutrition were estimated by recording the number of days the infants were exposed to each. Infants were first introduced to parenteral nutrition without lipid supplementation, soon followed within a day by introduction of intravenous fat emulsion (Intralipid, Baxter Healthcare Corporation,

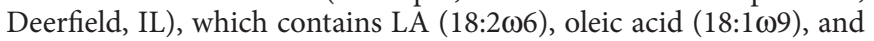

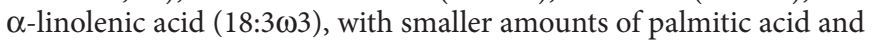
stearic acid (18:0). Intravenous nutrition was weaned off with tolerance for enteral feeding. Enteral feeding was occasionally supplemented for protein, but not lipids. Breast milk was used whenever available, otherwise formula (Similac Special Care, Abbott Nutrition, Abbott Park, IL) was used.

\section{MRI Studies}

MRI studies were performed on 1.5-T MRI scanners (General Electric Sigma, GE Medical Systems, Milwaukee, WI, or Siemens Avanto, Siemens Medical Solutions USA, Malvern, PA), soon after birth as clinically stable and again near near-term age. Conventional MRI sequences at both study sites were obtained as previously described, including T2-weighted and volumetric three-dimensional T1-weighted sequences (36). Scans were graded by a single neuroradiologist at each site (KJP, AJB) blinded to patient history for brain injury, including IVH, WMI, and cerebellar hemorrhage. IVH was scored using the grading system of Papile et al (2). WMI was scored using a system shown to be associated with neurodevelopmental outcomes at 12-18 mo, demonstrated to have good interrater reliability between these two neuroradiologists $(3,37)$. Injury severity was graded as the highest score from both scans.

Diffusion tensor imaging sequences were acquired using comparable $(4,7)$ multirepetition, single-shot echo planar sequences (UCSF 6 gradient directions; TR, 7s; TE, $100 \mathrm{~ms}$; slice thickness, $3 \mathrm{~mm}$ and 
UBC 12 gradient directions; TR, $4900 \mathrm{~ms}$; TE, $104 \mathrm{~ms}$; FOV, $160 \mathrm{~ms}$; slice thickness, $3 \mathrm{~mm})$ to determine FA and eigenvalues $(\lambda 1, \lambda 2$, and $\lambda 3$ ) in three dimensions describing axial $(\lambda 1)$, radial diffusivity (mean of $\lambda 2$ and $\lambda 3$ ), and mean diffusivity (mean of $\lambda 1, \lambda 2$, and $\lambda 3$ ) (27). FA describes the directionality of water diffusion. Axial diffusivity has been used as a measure of organization of axonal tracts (38), while radial diffusivity has been used as a measure of the quality of myelination (39). Data were analyzed blinded for patient history.

\section{Blood Samples}

Two blood samples were each drawn as close in time to each MRI scan as possible, timed with clinically indicated bloodwork and within a week of MRI. Samples were separated into plasma and red blood cell fractions and stored at $-70{ }^{\circ} \mathrm{C}$ until analysis. Proportions of each fatty acid relative to total fatty acids in the red cell membrane were determined by gas chromatography-flame ionization detector (17). Analysis was then focused to key PUFAs for their impact on brain development, including the $\omega$-3 fatty acids DHA (22:6 $\omega 3)$ and

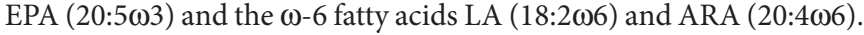

\section{Neurodevelopmental Outcome}

Infants were reassessed between 30-36 mo corrected age by a developmental psychologist or physiotherapist blinded to the child's neonatal course. Standardized assessment was performed using the Bayley Scales of Infant Development, 3rd Edition (Bayley-3), and composite cognitive, language, and motor scores were assigned based on the child's corrected age. Testing was performed in English or Spanish as appropriate. The Bayley- 3 has a mean of each composite score of 100 and a SD of 15 . Socioeconomic status variables were collected at follow-up from parental self-report, including total years of maternal education, maternal age at childbirth, race, and ethnicity. Race and ethnicity are defined as per the policies of the National Institutes of Health, where ethnicity includes those who are or are not Hispanic/ Latino, and race includes American Indian/Alaskan Native, Asian, Black/African American, Native Hawaiian/Other Pacific Islander, or White.

\section{Statistical Analysis}

Statistical analysis was performed using $\mathrm{R}$ version 3.1.1 (The $\mathrm{R}$ Foundation for Statistical Computing 2014, Vienna, Austria). All analyses were adjusted for study site to account for site-specific variations. To study the association between fatty acid levels and measures of brain injury (including IVH, WMI, and cerebellar hemorrhage), logistic regression analysis was used to adjust for known clinical risk factors for brain injury, including GA at birth (also associated with fatty acid levels) (10), chorioamnionitis (clinically suspected or confirmed), antenatal glucocorticoid exposure, 5-min Apgar score, patent ductus arteriosus (diagnosed by echocardiogram, with or without intervention), hypotension requiring medical intervention, intubation, and neonatal sepsis (presumed or confirmed, including necrotizing enterocolitis). Statistical significance was set at $P<0.05$.

Tract-based spatial statistics (FMRIB Software Library version 5.0, Oxford, UK) were used to assess potential associations between diffusion tensor imaging parameters and fatty acid levels in early and near-term blood samples. Data from all infants were fitted into a common space using nonlinear registration, allowing for voxelwise crosssubject statistical analysis of the white matter tract skeleton, using a previously validated method for this age group (40). Adjusting for age at time of scan, associations were considered significant at $P<0.05$.

Regions of interest were then selected from the PLIC and OR to compliment the findings from tract-based spatial statistics, using a method previously described (27). These regions encompass functionally relevant pathways and can be defined with high fidelity (4). The left and right hemispheric measurements were averaged for each region, as previous (4). To study the association between fatty acid levels and diffusion tensor imaging measures, linear mixed regression models were used to account for repeated measures. Adjustments were made for confounding factors selected a priori based on the previous studies (4) and limited due to sample size limitations, including IVH severity, WMI severity, patent ductus arteriosus, sepsis, and duration of intubation. Statistical significance was set at $P<0.05$.
Association between fatty acid level and outcome was explored using linear regression analysis to determine the long-term significance of fatty acid and imaging findings. Further adjustment for brain injury severity was performed to assess its contribution to the relationship. To understand how socioeconomic status may influence the association between fatty acid level and outcomes, linear regression analysis was performed.

\section{ACKNOWLEDGMENTS}

We would like to thank Kenneth J. Poskitt for his assistance reviewing MRI scans at the University of British Columbia study site.

\section{STATEMENT OF FINANCIAL SUPPORT}

This work is supported by the Gerber Foundation (Fremont, MI) (EWYT,DMF), Canadian Institutes for Health Research (CIHR) CHI 151135 (SPM), National Institutes of Health (NIH) R01 NS346432 (AJB), NIH P01NS082330 (DMF), NIH R01EB009756 (DX), NIH R01HD072074 (DX), and NeuroDevNet (Vancouver, Canada) (SPM).

Disclosure: Authors have no financial or other conflicts of interest to declare.

\section{REFERENCES}

1. Adams-Chapman I, Hansen NI, Stoll BJ, Higgins R; NICHD Research Network. Neurodevelopmental outcome of extremely low birth weight infants with posthemorrhagic hydrocephalus requiring shunt insertion. Pediatrics 2008;121:e1167-77.

2. Papile LA, Burstein J, Burstein R, Koffler H. Incidence and evolution of subependymal and intraventricular hemorrhage: a study of infants with birth weights less than 1,500 gm. J Pediatr 1978;92:529-34.

3. Miller SP, Ferriero DM, Leonard C, et al. Early brain injury in premature newborns detected with magnetic resonance imaging is associated with adverse early neurodevelopmental outcome. J Pediatr 2005;147:609-16.

4. Chau V, Poskitt KJ, McFadden DE, et al. Effect of chorioamnionitis on brain development and injury in premature newborns. Ann Neurol 2009;66:155-64.

5. Tam EW, Rosenbluth G, Rogers EE, et al. Cerebellar hemorrhage on magnetic resonance imaging in preterm newborns associated with abnormal neurologic outcome. J Pediatr 2011;158:245-50.

6. Limperopoulos C, Bassan H, Gauvreau K, et al. Does cerebellar injury in premature infants contribute to the high prevalence of long-term cognitive, learning, and behavioral disability in survivors? Pediatrics 2007;120: 584-93.

7. Bonifacio SL, Glass HC, Chau V, et al. Extreme premature birth is not associated with impaired development of brain microstructure. J Pediatr 2010;157:726-32.e1.

8. Lauritzen L, Hansen HS, Jørgensen MH, Michaelsen KF. The essentiality of long chain n-3 fatty acids in relation to development and function of the brain and retina. Prog Lipid Res 2001;40:1-94.

9. Martin CR, Dasilva DA, Cluette-Brown JE, et al. Decreased postnatal docosahexaenoic and arachidonic acid blood levels in premature infants are associated with neonatal morbidities. J Pediatr 2011;159:743-749.e1-2.

10. Agostoni C, Marangoni F, Stival G, et al. Whole blood fatty acid composition differs in term versus mildly preterm infants: small versus matched appropriate for gestational age. Pediatr Res 2008;64:298-302.

11. Feng Z, Zou X, Jia H, et al. Maternal docosahexaenoic acid feeding protects against impairment of learning and memory and oxidative stress in prenatally stressed rats: possible role of neuronal mitochondria metabolism. Antioxid Redox Signal 2012;16:275-89.

12. Crawford MA, Golfetto I, Ghebremeskel K, et al. The potential role for arachidonic and docosahexaenoic acids in protection against some central nervous system injuries in preterm infants. Lipids 2003;38:303-15.

13. Martinez M, Vazquez E. MRI evidence that docosahexaenoic acid ethyl ester improves myelination in generalized peroxisomal disorders. Neurology 1998;51:26-32.

14. Calderon F, Kim HY. Docosahexaenoic acid promotes neurite growth in hippocampal neurons. J Neurochem 2004;90:979-88.

15. Martin RE, Bazan NG. Changing fatty acid content of growth cone lipids prior to synaptogenesis. J Neurochem 1992;59:318-25. 


\section{Articles | Tametal.}

16. Beyerlein A, Hadders-Algra M, Kennedy K, et al. Infant formula supplementation with long-chain polyunsaturated fatty acids has no effect on Bayley developmental scores at 18 months of age-IPD meta-analysis of 4 large clinical trials. J Pediatr Gastroenterol Nutr 2010;50:79-84.

17. Innis SM, Nelson CM, Rioux MF, King DJ. Development of visual acuity in relation to plasma and erythrocyte omega- 6 and omega- 3 fatty acids in healthy term gestation infants. Am J Clin Nutr 1994;60:347-52.

18. Tanaka K, Kon N, Ohkawa N, Yoshikawa N, Shimizu T. Does breastfeeding in the neonatal period influence the cognitive function of very-low-birthweight infants at 5 years of age? Brain Dev 2009;31:288-93.

19. Clandinin MT, Van Aerde JE, Merkel KL, et al. Growth and development of preterm infants fed infant formulas containing docosahexaenoic acid and arachidonic acid. J Pediatr 2005; 146:461-8.

20. O'Connor DL, Hall R, Adamkin D, et al.; Ross Preterm Lipid Study. Growth and development in preterm infants fed long-chain polyunsaturated fatty acids: a prospective, randomized controlled trial. Pediatrics 2001;108:359-71.

21. Makrides M, Gibson RA, McPhee AJ, et al. Neurodevelopmental outcomes of preterm infants fed high-dose docosahexaenoic acid: a randomized controlled trial. JAMA 2009;301:175-82.

22. Volpe JJ. Neurology of the Newborn. 5th edn. Philadelphia, PA: Saunders, 2008.

23. Bazan NG, Molina MF, Gordon WC. Docosahexaenoic acid signalolipidomics in nutrition: significance in aging, neuroinflammation, macular degeneration, Alzheimer's, and other neurodegenerative diseases. Annu Rev Nutr 2011;31:321-51.

24. Stillwell W, Wassall SR. Docosahexaenoic acid: membrane properties of a unique fatty acid. Chem Phys Lipids 2003;126:1-27.

25. Alderliesten T, Lemmers PM, Smarius JJ, van de Vosse RE, Baerts W, van Bel F. Cerebral oxygenation, extraction, and autoregulation in very preterm infants who develop peri-intraventricular hemorrhage. J Pediatr 2013;162:698-704.e2.

26. Milligan DW. Failure of autoregulation and intraventricular haemorrhage in preterm infants. Lancet 1980;1:896-8.

27. Partridge SC, Mukherjee P, Henry RG, et al. Diffusion tensor imaging: serial quantitation of white matter tract maturity in premature newborns. Neuroimage 2004;22:1302-14.

28. Mukherjee P, Miller JH, Shimony JS, et al. Diffusion-tensor MR imaging of gray and white matter development during normal human brain maturation. AJNR Am J Neuroradiol 2002;23:1445-56.
29. Grayson DS, Kroenke CD, Neuringer M, Fair DA. Dietary omega-3 fatty acids modulate large-scale systems organization in the rhesus macaque brain. J Neurosci 2014;34:2065-74.

30. Simmer K, Rao SC. Early introduction of lipids to parenterally-fed preterm infants. Cochrane Database Syst Rev 2005:CD005256

31. Pinto TJ, Farias DR, Rebelo F, et al. Lower inter-partum interval and unhealthy life-style factors are inversely associated with n-3 essential fatty acids changes during pregnancy: a prospective cohort with Brazilian women. PLoS One 2015;10:e0121151.

32. Glew RH, Wold RS, Herbein JH, Wark WA, Martinez MA, Vanderjagt DJ. Low docosahexaenoic acid in the diet and milk of women in New Mexico. J Am Diet Assoc 2008;108:1693-9.

33. Lora KR, Lewis NM, Eskridge KM, Stanek-Krogstrand K, Travnicek DA. Correlation of omega-3 fatty acids intakes with acculturation and socioeconomic status in midwestern Latinas. J Immigr Minor Health 2011;13: $111-8$.

34. Birch EE, Carlson SE, Hoffman DR, et al. The DIAMOND (DHA Intake And Measurement Of Neural Development) Study: a double-masked, randomized controlled clinical trial of the maturation of infant visual acuity as a function of the dietary level of docosahexaenoic acid. Am J Clin Nutr 2010;91:848-59.

35. Létondor A, Buaud B, Vaysse C, et al. Erythrocyte DHA level as a biomarker of DHA status in specific brain regions of $n-3$ long-chain PUFAsupplemented aged rats. Br J Nutr 2014;112:1805-18.

36. Tam EW, Miller SP, Studholme C, et al. Differential effects of intraventricular hemorrhage and white matter injury on preterm cerebellar growth. J Pediatr 2011;158:366-71.

37. Chau V, Synnes A, Grunau RE, Poskitt KJ, Brant R, Miller SP. Abnormal brain maturation in preterm neonates associated with adverse developmental outcomes. Neurology 2013;81:2082-9.

38. Choe AS, Stepniewska I, Colvin DC, Ding Z, Anderson AW. Validation of diffusion tensor MRI in the central nervous system using light microscopy: quantitative comparison of fiber properties. NMR Biomed 2012;25:900-8.

39. Tuor UI, Morgunov M, Sule M, et al. Cellular correlates of longitudinal diffusion tensor imaging of axonal degeneration following hypoxic-ischemic cerebral infarction in neonatal rats. Neuroimage Clin 2014;6:32-42.

40. Duerden EG, Foong J, Chau V, et al. Tract-based spatial statistics in preterm-born neonates predicts cognitive and motor outcomes at 18 months. AJNR Am J Neuroradiol 2015;36:1565-71. 